\title{
Toeplitz operators with distributional symbols on Bergman spaces
}

Article

Published Version

Perälä, A., Taskinen, J. and Virtanen, J. (2011) Toeplitz operators with distributional symbols on Bergman spaces. Proceedings of the Edinburgh Mathematical Society, 54 (02). pp. 505-514. ISSN 1464-3839 doi:

https://doi.org/10.1017/S001309151000026X Available at https://centaur.reading.ac.uk/29124/

It is advisable to refer to the publisher's version if you intend to cite from the work. See Guidance on citing.

To link to this article DOI: http://dx.doi.org/10.1017/S001309151000026X

Publisher: Edinburgh Mathematical Society

All outputs in CentAUR are protected by Intellectual Property Rights law, including copyright law. Copyright and IPR is retained by the creators or other copyright holders. Terms and conditions for use of this material are defined in the End User Agreement.

\section{www.reading.ac.uk/centaur}

\section{CentAUR}

Central Archive at the University of Reading 
Reading's research outputs online 


\title{
TOEPLITZ OPERATORS WITH DISTRIBUTIONAL SYMBOLS ON BERGMAN SPACES
}

\author{
ANTTI PERÄL $\ddot{A}^{1}$, JARI TASKINEN ${ }^{1}$ AND JANI VIRTANEN ${ }^{2}$ \\ ${ }^{1}$ Department of Mathematics, University of Helsinki, 00014 Helsinki, \\ Finland (antti.i.perala@helsinki.fi; jari.taskinen@helsinki.fi) \\ ${ }^{2}$ Courant Institute of Mathematical Sciences, New York University, \\ 251 Mercer Street, New York, NY 10012, USA (virtanen@courant.nyu.edu)
}

(Received 1 February 2010)

\begin{abstract}
We study the boundedness and compactness of Toeplitz operators $T_{a}$ on Bergman spaces $A^{p}(\mathbb{D}), 1<p<\infty$. The novelty is that we allow distributional symbols. It turns out that the belonging of the symbol to a weighted Sobolev space $W_{\nu}^{-m, \infty}(\mathbb{D})$ of negative order is sufficient for the boundedness of $T_{a}$. We show the natural relation of the hyperbolic geometry of the disc and the order of the distribution. A corresponding sufficient condition for the compactness is also derived.
\end{abstract}

Keywords: Toeplitz operator; Bergman space; distribution; bounded operator; compact operator

2010 Mathematics subject classification: Primary 47B35

\section{Introduction}

The aim of this work is to find a general class of distributions $a$ on the unit disc $\mathbb{D}$ of the complex plane $\mathbb{C}$, such that a Toeplitz operator with symbol $a$ becomes well defined and bounded on (reflexive) Bergman spaces. We provide a sufficient condition for boundedness, which involves a natural connection of the order or singularity of the distribution on one hand, and of the hyperbolic geometry of $\mathbb{D}$ on the other. Roughly, if the symbol $a$ is a $k$ th-order distributional derivative of a bounded function $b: \mathbb{D} \rightarrow \mathbb{C}$, then the function $b(z) \nu(z)^{-k}:=b(z)\left(1-|z|^{2}\right)^{-k}$ should be bounded (Theorem 3.1). More precisely, the sufficient condition is expressed in terms of $a$ belonging to a weighted Sobolev space (see Definition 2.3). The corresponding sufficient condition for compactness is given in Theorem 4.2 .

Consider the space $L^{p}:=L^{p}(\mathbb{D}), 1 \leqslant p \leqslant \infty$, defined using the normalized area measure $\mathrm{d} A$ on $\mathbb{D}$ and the Bergman space $A^{p}$, which is the closed subspace of $L^{p}$ consisting of analytic functions. The Bergman projection $P$ is the orthogonal projection of $L^{2}$ onto $A^{2}$, and it has the integral representation

$$
P f(z)=\int_{\mathbb{D}} \frac{f(\zeta)}{(1-z \bar{\zeta})^{2}} \mathrm{~d} A(\zeta)
$$

(c) 2011 The Edinburgh Mathematical Society 
It is also known to be a bounded projection of $L^{p}$ onto $A^{p}$ when $1<p<\infty$. For an integrable function $a: \mathbb{D} \rightarrow \mathbb{C}$ and, say, bounded analytic functions $f$, the Toeplitz operator $T_{a}$ with symbol $a$ is defined by setting

$$
T_{a} f=P(a f) .
$$

Since $P$ is bounded, it easily follows that $T_{a}$ extends to a bounded operator $A^{p} \rightarrow A^{p}$ for $1<p<\infty$, whenever $a$ is a bounded measurable function. A more general sufficient condition for the boundedness of $T_{a}$ was recently given in [9]. The condition is a rather weak requirement of the boundedness of certain 'averages' of $a$ over hyperbolic rectangles [9, Theorem 2.3].

As for distributional symbols, if $a$ is a compactly supported distribution on $\mathbb{D}$, one can directly define the corresponding Toeplitz operator by

$$
T_{a} f(z)=\left\langle f(\zeta)(1-z \bar{\zeta})^{-2}, a\right\rangle_{\zeta}
$$

where $\langle\cdot, \cdot\rangle_{\zeta}$ denotes the dual pairing of the test function and distribution spaces and the test function is considered as a function of the variable $\zeta$, with $z$ being a parameter. However, this definition is not satisfactorily general, since $L^{1}$-functions that are compactly supported distributions are also compactly supported as functions. Below we give a more general definition for distributional symbols, the supports of which do not have any artificial restrictions, and the order of which may be arbitrary, though finite.

Obviously, our sufficient condition for boundedness seems quite different from the one in $[\mathbf{9}]$, although both conditions are related to the hyperbolic geometry. The exact relation of both conditions remains an open problem; the same holds, of course, for the problem of how close any of the conditions is to also being necessary.

For an account of recent developments in the study of boundedness of Toeplitz operators, see [7]. Distribution symbols were used in [2], but for different purposes than in the present work. Other related works are $[\mathbf{3}],[\mathbf{5}],[\mathbf{8}]$ (for the case $p=1$ ), $[\mathbf{1 1}],[\mathbf{1 2}]$ and $[\mathbf{1 4}]$ (see also the references in $[\mathbf{7}, \mathbf{1 0}]$ ).

\section{Notation and terminology}

We follow the definitions and terminology used in $[\mathbf{4 , 6}]$ for general theory of distributions. For Sobolev spaces, we refer the reader to [1]. For operator theory and analytic function spaces, in particular Bergman spaces, see [13]. By $C, C^{\prime}, C_{1}, c$, etc. (respectively, $C_{n}$, etc.), we denote positive constants independent of functions, variables or indices occurring in the given calculations (respectively, depending only on $n$ ). These may vary from place to place, but not in the same group of inequalities. For the norm of an element $f$ of a Banach function space $X$ we use the notation $\|f ; X\|$. For the operator norm of a bounded linear operator $T: X \rightarrow Y$, we write $\|T: X \rightarrow Y\|$. In what follows we consider various function and distribution spaces, all of which are defined on $\mathbb{D}$ unless otherwise stated. The standard space of distributions on the disc is denoted by $\mathcal{D}^{\prime}=\mathcal{D}^{\prime}(\mathbb{D})$. The order of a multi-index $\alpha \in \mathbb{N}^{2}$, where $\mathbb{N}:=\{0,1,2, \ldots\}$, is denoted by 
$|\alpha|:=\alpha_{1}+\alpha_{2}$. The notation $\alpha \geqslant \beta$ for the multi-indices $\alpha, \beta$ means that $\alpha_{j} \geqslant \beta_{j}$ for $j=1,2$. For derivatives, the notation $D^{\alpha} f$ stands for

$$
\frac{\partial^{\alpha_{1}}}{\partial x^{\alpha_{1}}} \frac{\partial^{\alpha_{2}}}{\partial y^{\alpha_{2}}} f
$$

if $f$ is a function of $z=x+\mathrm{i} y$, where $x, y \in \mathbb{R}$, and $\alpha$ is a multi-index. The same notation is used for both classical and distributional derivatives. We also write $D_{\zeta}^{\alpha} f$ if it is necessary to indicate the same differentiation of a function $f$ with respect to its variable $\zeta$. For an analytic function $f$, we denote by $f^{(l)}$ the $l$ th derivative with respect to $z \in \mathbb{C}$ for all $l \in \mathbb{N}$.

\section{Weighted Sobolev spaces}

We define the standard weight function $\nu: \mathbb{D} \rightarrow \mathbb{R}^{+}$by

$$
\nu(z)=1-|z|^{2}
$$

Given $m \in \mathbb{N}$, we denote by $W_{\nu}^{m, 1}:=W_{\nu}^{m, 1}(\mathbb{D})$ the weighted Sobolev space consisting of measurable functions $f$ on $\mathbb{D}$ such that the distributional derivatives satisfy

$$
\begin{aligned}
\left\|f ; W_{\nu}^{m, 1}\right\| & :=\sum_{|\alpha| \leqslant m}\left\|D^{\alpha} f(z) ; L_{\nu|\alpha|}^{1}\right\| \\
& :=\sum_{|\alpha| \leqslant m} \int_{\mathbb{D}}\left|D^{\alpha} f(z)\right| \nu(z)^{|\alpha|} \mathrm{d} A(z) \\
r & <\infty .
\end{aligned}
$$

Remark 2.1. For readers who are unfamiliar with the details of Sobolev spaces we recall that the space $C_{0}^{\infty}(\mathbb{D})$ of compactly supported infinitely smooth functions on the disc is not dense in the unweighted Sobolev space $W^{m, 1}(\mathbb{D})[\mathbf{1}$, Chapter 3]. This unpleasant fact complicates the treatment of the dual space.

However, the following fact holds in the weighted case. The result is known, but we sketch the proof for the convenience of the reader.

Lemma 2.2. The subspace $C_{0}^{\infty}:=C_{0}^{\infty}(\mathbb{D})$ is dense in $W_{\nu}^{m, 1}$.

Proof. First we remark that if the support of a $g \in W_{\nu}^{m, 1}(\mathbb{D})$ is contained in a compact $\operatorname{disc} \Omega_{r}:=\{|z| \leqslant r\}$ with $0<r<1$, then it can be approximated in $W_{\nu}^{m, 1}(\mathbb{D})$ by an element of $C_{0}^{\infty}(\mathbb{D})$. This follows from [1, Lemma 3.15] by choosing the set $\Omega^{\prime}$ there to be a disc $\Omega_{s}$ with $s>r$. The convergence $\lim _{\varepsilon \rightarrow 0} J_{\varepsilon} * g=g$ in $W^{m, 1}\left(\Omega^{\prime}\right)$ (using the notation of the citation) also implies the convergence in $W_{\nu}^{m, 1}(\mathbb{D})$.

Consequently, it suffices to approximate an arbitrary $f \in W_{\nu}^{m, 1}$ by a compactly supported element of $W_{\nu}^{m, 1}$. To this end, note that it is possible to define a sequence of radial cut-off functions $\chi_{n} \in C_{0}^{\infty}, n=4,5,6, \ldots$, such that $\chi_{n}(z)=\chi_{n}(|z|)$ for $z \in \mathbb{D}$, 
$0 \leqslant \chi_{n}(r) \leqslant 1$ for all $0 \leqslant r<1, \chi_{n}(r)=1$ for $0 \leqslant r \leqslant 1-3 / n, \chi_{n}(r)=0$ for $1-1 / n \leqslant r \leqslant 1$, and such that, for all $k \in \mathbb{N}$,

$$
\frac{\mathrm{d}^{k} \chi_{n}(r)}{\mathrm{d} r^{k}} \mid \leqslant C_{k} n^{k}
$$

for all $0<r<1$. In fact, we can define $\chi_{n}$ as the usual convolution

$$
\chi_{n}(r)=\int_{-\infty}^{\infty} X_{[-1+2 / n, 1-2 / n]}(\varrho) J_{n}(r-\varrho) \mathrm{d} \varrho,
$$

where $X_{[-1+2 / n, 1-2 / n]}$ is the characteristic function of the interval $[-1+2 / n, 1-2 / n]$ and $J_{n}$ is the standard mollifier

$$
J_{n}(r)= \begin{cases}C n \mathrm{e}^{-1 /\left(1-(n r)^{2}\right)} & \text { if }|r| \leqslant 1 / n, \\ 0 & \text { if }|r|>1 / n,\end{cases}
$$

and $C>0$ is a constant independent of $n$ making the integral

$$
\int_{-\infty}^{\infty} J_{n} \mathrm{~d} r
$$

equal to 1 . The property (2.3) now follows by differentiating $J_{n}$ under the integral sign in (2.4).

A good approximation of a given $f \in W_{\nu}^{m, p}$ is then $\chi_{n} f$ for a sufficiently large $n$. To see this, in the case $m=1$, for example, we define $D_{n}:=\{z|| z \mid \geqslant 1-3 / n\}$ and estimate one of the terms $(\alpha=(1,0))$ in $(2.2)$ as

$$
\begin{aligned}
\int_{\mathbb{D}}\left|\frac{\partial}{\partial x}\left(f-\chi_{n} f\right)\right| \nu \mathrm{d} A & \leqslant \int_{\mathbb{D}}\left|\frac{\partial f}{\partial x}\right|\left|1-\chi_{n}\right| \nu \mathrm{d} A+\int_{\mathbb{D}}|f|\left|\frac{\partial \chi_{n}}{\partial x}\right| \nu \mathrm{d} A \\
& \leqslant C \int_{D_{n}}\left|\frac{\partial f}{\partial x}\right| \nu \mathrm{d} A+C \int_{D_{n}}|f| n\left(1-\left(1-\frac{3}{n}\right)\right) \mathrm{d} A \\
& =C \int_{D_{n}}\left|\frac{\partial f}{\partial x}\right| \nu \mathrm{d} A+C^{\prime} \int_{D_{n}}|f| \mathrm{d} A,
\end{aligned}
$$

where we used the facts that $\partial \chi_{n} / \partial x$ vanishes outside $D_{n}$ and $\left|\partial \chi_{n} / \partial x\right| \leqslant C n$ on $D_{n}$ by (2.3). Now (2.5) approaches 0 as $n \rightarrow \infty$, since the area of $D_{n}$ tends to 0 and the integrals

$$
\int_{\mathbb{D}}|f| \mathrm{d} A \text { and } \int_{\mathbb{D}}\left|\frac{\partial f}{\partial x}\right| \nu \mathrm{d} A
$$

are bounded (by $\left\|f ; W_{\nu}^{m, 1}\right\|$ ). The other terms in (2.2) have similar or easier estimates, which proves the lemma for $m=1$.

The idea for the higher $m$ is similar: the higher powers of $\nu$ in (2.2) cancel out the growth of higher derivatives in (2.3).

As a consequence of this, it is possible to describe the dual space. 
Definition 2.3. Given $m \in \mathbb{N}$ we denote by $W_{\nu}^{-m, \infty}:=W_{\nu}^{-m, \infty}(\mathbb{D})$ the (weighted Sobolev) space consisting of distributions $a$ on $\mathbb{D}$, which can be written in the form

$$
a=\sum_{0 \leqslant|\alpha| \leqslant m}(-1)^{|\alpha|} D^{\alpha} b_{\alpha}
$$

where

$$
b_{\alpha} \in L_{\nu^{-|\alpha|}}^{\infty}:=L_{\nu^{-|\alpha|}}^{\infty}(\mathbb{D})
$$

i.e.

$$
\left\|b_{\alpha} ; L_{\nu^{-|\alpha|} \mid}^{\infty}\right\|:=\underset{\mathbb{D}}{\operatorname{ess} \sup } \nu(z)^{-|\alpha|}\left|b_{\alpha}(z)\right|<\infty .
$$

Here every $b_{\alpha}$ is considered as a distribution like a locally integrable function, and the identity (2.6) contains distributional derivatives.

Given such an $a$, the representation (2.6) is not unique in general. Hence, we define the norm of $a$ by

$$
\|a\|:=\left\|a ; W_{\nu}^{-m, \infty}\right\|:=\inf \max _{0 \leqslant|\alpha| \leqslant m}\left\|b_{\alpha} ; L_{\nu^{-|\alpha|} \mid}^{\infty}\right\|,
$$

where the infimum is taken over all representations (2.6).

Lemma 2.4. The dual of $W_{\nu}^{m, 1}$ is isometrically isomorphic to $W_{\nu}^{-m, \infty}$ with respect to the dual pairing

$$
\langle f, a\rangle:=\sum_{0 \leqslant|\alpha| \leqslant m} \int_{\mathbb{D}}\left(D^{\alpha} f\right) b_{\alpha} \mathrm{d} A,
$$

where $f \in W_{\nu}^{m, 1}, a \in W_{\nu}^{-m, \infty}$ and the functions $b_{\alpha}$ are as in (2.6).

Proof. First, define the Banach space products

$$
X:=\prod_{|\alpha| \leqslant m} L_{\nu|\alpha|}^{1}(\mathbb{D}), \quad Y:=\prod_{|\alpha| \leqslant m} L_{\nu^{-|\alpha|}}^{\infty}(\mathbb{D})
$$

which have the norms

$$
\|f ; X\|=\sum_{|\alpha| \leqslant m}\left\|f_{\alpha} ; L_{\nu|\alpha|}^{1}\right\|
$$

and

$$
\|g ; Y\|=\max _{|\alpha| \leqslant m}, \quad\left\|g_{\alpha} ; L_{\nu^{-}|\alpha|}^{\infty}\right\|,
$$

where $f=\left(f_{\alpha}\right) \in X$ and $g=\left(g_{\alpha}\right) \in Y$. Since $L_{\nu^{-|\alpha|}}^{\infty}(\mathbb{D})$ is the dual of $L_{\nu^{|\alpha|}}^{1}(\mathbb{D})$ with respect to the standard dual pairing

$$
\langle h, k\rangle=\int_{\mathbb{D}} h k \mathrm{~d} A
$$


$Y$ is also the dual of $X$ with respect to the pairing

$$
\langle f, a\rangle:=\sum_{0 \leqslant|\alpha| \leqslant m} \int_{\mathbb{D}} f_{\alpha} g_{\alpha} \mathrm{d} A
$$

and the dual norm of $X^{*}$ coincides with the norm of $Y$. This is well known, but it can also be proven following the argument of $[\mathbf{1},(3.7)]$.

Next we claim that, for every element $L$ in the dual space $\left(W_{\nu}^{m, 1}\right)^{*}$, there exists an element $g=\left(g_{\alpha}\right) \in Y$ such that

$$
L(f)=\sum_{0 \leqslant|\alpha| \leqslant m} \int_{\mathbb{D}}\left(D^{\alpha} f\right) g_{\alpha} \mathrm{d} A
$$

for all $f \in W_{\nu}^{m, 1}$. Moreover, $\left\|L ; X^{*}\right\|=\inf \|g ; Y\|$, where the infimum is taken over all $g=\left(g_{\alpha}\right)$ such that the above identity holds.

The proof of these facts follows that of [1, Theorem 3.8]. First, the operator $P: W_{\nu}^{m, 1} \rightarrow$ $X, P f=\left(D^{\alpha} f\right)$ is an isometry. Defining $W:=P\left(W_{\nu}^{m, 1}\right) \subset X$, a linear functional $L^{*}$ is defined on $W$ by

$$
L^{*}(P f)=L(f), \quad f \in W_{\nu}^{m, 1} .
$$

Since $P$ is isometric, $L^{*} \in W^{*}$ and $\left\|L^{*} ; W^{*}\right\|=\left\|L ;\left(W_{\nu}^{m, 1}\right)^{*}\right\|$. We denote by $\tilde{L}$ the HahnBanach extension of $L^{*}$ to all of $X$ and, using the above described duality, we find a $g=\left(g_{\alpha}\right) \in Y$ such that

$$
\tilde{L}(f)=\sum_{0 \leqslant|\alpha| \leqslant m} \int_{\mathbb{D}} f_{\alpha} g_{\alpha} \mathrm{d} A \text { for } f=\left(f_{\alpha}\right) \in X .
$$

Thus, if $f \in W_{\nu}^{m, 1}$, then

$$
L(f)=L^{*}(P f)=\tilde{L}(P f)=\sum_{0 \leqslant|\alpha| \leqslant m} \int_{\mathbb{D}}\left(D^{\alpha} f\right) g_{\alpha} \mathrm{d} A
$$

and, moreover, $\left\|L ;\left(W_{\nu}^{m, 1}\right)^{*}\right\|=\|g ; Y\|$.

If $h \in Y$ is another element such that (2.14) holds, it corresponds to an extension of $L^{*}$, and thus $\|h ; Y\|$ is at least $\left\|L ;\left(W_{\nu}^{m, 1}\right)^{*}\right\|$. This completes the proof of the claims.

Assume that $L \in\left(W_{\nu}^{m, 1}\right)^{*}$ and the corresponding $g \in Y$ are given. We observe that $L$ is an extension to $W_{\nu}^{m, 1}$ of the distribution

$$
T=\sum_{|\alpha| \leqslant m}(-1)^{|\alpha|} D^{\alpha} g_{\alpha}
$$

where the derivatives are distributional and $g_{\alpha}$ is the distribution

$$
\varphi \mapsto \int_{\mathbb{D}} g_{\alpha} \varphi \mathrm{d} A
$$

for the test functions $\varphi$. This follows directly by applying (2.14) and (2.15) to an arbitrary test function. Finally, by Lemma 2.2, the extension of (2.15) to $W_{\nu}^{m, 1}$ is unique (for details of this argument, see the proof of Theorem 3.9 of [1] ). The correspondence of $L$ and this unique extension of $T$ establish the statement of the lemma. 
Remark 2.5. Let $a \in W_{\nu}^{-m, \infty}$ be given. Although the representation (2.6) is not unique, the value of the expression on the right-hand side of (2.10) is. This follows from the duality theory presented above.

\section{Boundedness of Toeplitz operators with distributional symbols}

In what follows, $a$ is a distribution on $\mathbb{D}$ having a finite order. Assuming $a$ belongs to a weighted Sobolev space described in the previous section, we give the definition of a Toeplitz operator with symbol $a$, and prove its boundedness on reflexive Bergman spaces. We remark that this result can be applied, for example, to all compactly supported distributions, but since such distributions actually determine compact operators, this case is only considered in the next section.

Theorem 3.1. Assume that the distribution $a \in \mathcal{D}^{\prime}$ belongs to $W_{\nu}^{-m, \infty}$ for some $m$. Then the Toeplitz operator $T_{a}$, defined by the formula

$$
T_{a} f(z)=\sum_{0 \leqslant|\alpha| \leqslant m} \int_{\mathbb{D}}\left(D_{\zeta}^{\alpha} \frac{f(\zeta)}{(1-z \bar{\zeta})^{2}}\right) b_{\alpha}(\zeta) \mathrm{d} A(\zeta), \quad f \in A^{p},
$$

is well defined and bounded $A^{p} \rightarrow A^{p}$ for all $1<p<\infty$. The resulting operator is independent of the choice of the representation (2.6). Moreover, there is a constant $C>0$ such that

$$
\left\|T_{a}: A^{p} \rightarrow A^{p}\right\| \leqslant C\left\|a ; W_{\nu}^{-m, \infty}\right\| .
$$

Proof. To prove the boundedness, let us fix a representation (2.6) such that

$$
\left\|a ; W_{\nu}^{-m, \infty}\right\| \geqslant \frac{1}{2} \max _{0 \leqslant|\alpha| \leqslant m}\left\|b_{\alpha} ; L_{\nu^{-|\alpha|}}^{\infty}\right\| .
$$

Since $2|1-z \bar{\zeta}| \geqslant 1-|\zeta|$ we can estimate, for every $f \in A^{p}$,

$$
\begin{aligned}
\sum_{0 \leqslant|\alpha| \leqslant m}\left|D_{\zeta}^{\alpha} \frac{f(\zeta)}{(1-z \bar{\zeta})^{2}}\right|\left|b_{\alpha}(\zeta)\right| & \\
& \leqslant C \sum_{|\alpha| \leqslant m} \sum_{\beta \leqslant \alpha}\left|\left(D^{\beta} f\right)(\zeta) D_{\zeta}^{\alpha-\beta}(1-z \bar{\zeta})^{-2}\right|\left|b_{\alpha}(\zeta)\right| \\
& \leqslant C_{1} \sum_{|\alpha| \leqslant m} \sum_{\beta \leqslant \alpha}\left|\left(D^{\beta} f\right)(\zeta)(1-z \bar{\zeta})^{-2-|\alpha|+|\beta|}\right|\left|b_{\alpha}(\zeta)\right| \\
& \leqslant C_{2} \sum_{|\alpha| \leqslant m} \sum_{\beta \leqslant \alpha}\left|\left(D^{\beta} f\right)(\zeta)(1-z \bar{\zeta})^{-2}\right|\left(1-|\zeta|^{2}\right)^{-|\alpha|+|\beta|}\left|b_{\alpha}(\zeta)\right| \\
& \leqslant C_{3}\left\|a ; W_{\nu}^{-m, \infty}\right\| \sum_{|\alpha| \leqslant m} \sum_{\beta \leqslant \alpha}\left|\left(D^{\beta} f\right)(\zeta)(1-z \bar{\zeta})^{-2}\right|\left(1-|\zeta|^{2}\right)^{|\beta|} \\
& \leqslant C_{4}\left\|a ; W_{\nu}^{-m, \infty}\right\| \sum_{j=0}^{m} \frac{\left|f^{(j)}(\zeta)\right|\left(1-|\zeta|^{2}\right)^{j}}{|1-z \bar{\zeta}|^{2}}
\end{aligned}
$$


Note that $\left|D^{\beta} f\right|=\left|f^{(|\beta|)}\right|$ for all analytic functions. We now recall that, given $g \in A^{p}$, the functions $\left|g^{(l)}(z)\right|\left(1-|z|^{2}\right)^{l}$ belong to $L^{p}$ with norms bounded by $C_{l}\|g\|_{p}$ [13, Theorem 4.28]. Moreover, the maximal Bergman projection is bounded on $L^{p}$, i.e. for some constant $C>0$,

$$
\left\|\int_{\mathbb{D}} \frac{|g(\zeta)|}{|1-z \bar{\zeta}|^{2}} \mathrm{~d} A(\zeta)\right\|_{p} \leqslant C\|g\|_{p}
$$

for all $g \in L^{p}[\mathbf{1 3}$, Corollary 3.13]. These facts, together with the definition (3.1) and the estimate (3.4), prove that $T f \in A^{p}$ with the norm estimate (3.2).

The uniqueness of the definition (3.1) is a direct consequence of Remark 2.5 as soon as we prove that, for all $f \in A^{p}$ and every fixed $z \in \mathbb{D}$, the function

$$
F_{z}(\zeta):=\frac{f(\zeta)}{(1-z \bar{\zeta})^{2}}
$$

of the variable $\zeta$ belongs to the Sobolev space $W_{\nu}^{m, 1}$. But this follows from the previously cited result that $\left|f^{(l)}(\zeta)\right|\left(1-|\zeta|^{2}\right)^{l} \in L^{p} \subset L^{1}$ for all $l \in \mathbb{N}$. We also obtain $\left|\left(D_{\zeta}^{\alpha} F_{z}\right)(\zeta)\right|\left(1-|\zeta|^{2}\right)^{|\alpha|} \in L^{1}$ for all $\alpha,|\alpha| \leqslant m$, since the factor $(1-z \bar{\zeta})^{-2}$ and all of its derivatives are bounded functions of $\zeta$ (for a fixed $z$ ).

A non-trivial example is the symbol

$$
a:=b_{(0,0)}+D^{(1,0)} b_{(1,0)}=b_{(0,0)}+\frac{\partial}{\partial x} b_{(1,0)},
$$

where, for $z=x+\mathrm{i} y=r \mathrm{e}^{\mathrm{i} \theta}$,

$$
b_{(0,0)}(z)= \begin{cases}0 & \text { if } x \leqslant 0 \\ 2 x & \text { if } x>0\end{cases}
$$

and

$$
b_{(1,0)}(z)= \begin{cases}0 & \text { if } x \leqslant 0 \\ 1-r^{2} & \text { if } x>0\end{cases}
$$

Note that $b_{(1,0)}$ can be written as the product $Y(x)\left(1-r^{2}\right)$, where $Y$ is the usual step function of one real variable. Denoting by $\delta_{0}(x)$ the Dirac measure of 0 with respect to the variable $x \in \mathbb{R}$, we get

$$
\begin{aligned}
a & =b_{(0,0)}+D^{(1,0)} b_{(1,0)} \\
& =b_{(0,0)}+\left(D^{(1,0)} Y(x)\right)\left(1-r^{2}\right)+Y(x) D^{(1,0)}\left(1-r^{2}\right) \\
& =\delta_{0}(x)\left(1-r^{2}\right) \\
& =\delta_{0}(x)\left(1-y^{2}\right),
\end{aligned}
$$

since $D^{(1,0)}\left(1-r^{2}\right)=-2 x$. The symbol $a$ is thus a weighted Dirac measure of the line segment $\{z \in \mathbb{D} \mid \operatorname{Re} z=0\}$. Clearly, $a \in W_{\nu}^{-1, \infty}$, and it hence defines a bounded Toeplitz operator $A^{p} \rightarrow A^{p}$. Note that the support of $a$ is not compact in $\mathbb{D}$. 


\section{Compactness of Toeplitz operators with distributional symbols}

We start with a remark on distributional symbols with compact supports.

Proposition 4.1. Any distribution $a \in \mathcal{D}^{\prime}$ with compact support belongs to the Sobolev space $W_{\nu}^{-m, \infty}$. Via the formula (3.1), it defines a Toeplitz operator that is a compact operator $A^{p} \rightarrow A^{p}$.

Proof of Proposition 4.1. A well-known basic result in distribution theory (see, for example, [6, Theorem 6.26]) states that any compactly supported distribution $a$ has a finite order $m$ and can be presented as

$$
a=\sum_{|\alpha| \leqslant m} D^{\alpha} b_{\alpha}
$$

where the functions $b_{\alpha}$ can be assumed to be continuous and supported in an arbitrary neighbourhood $V$ of $\operatorname{supp} a$. In particular, we may assume that the closure $\bar{V}$ is a compact subset of $\mathbb{D}$. Trivially, such functions $b_{\alpha}$ satisfy (2.8); hence, $a \in W_{\nu}^{-m, \infty}$. Also, the compactness of the supports of $b_{\alpha}$ implies that the operator (3.1) is compact on $A^{p}$.

Theorem 4.2. Assume that the symbol $a \in \mathcal{D}^{\prime}$ belongs to $W_{\nu}^{-m, \infty}$ for some $m$. The Toeplitz operator $T_{a},(3.1)$, is compact if a has a representation (2.6) such that the functions $b_{\alpha}$ satisfy

$$
\lim _{r \rightarrow 1} \operatorname{ess} \sup \nu(z)^{-|\alpha|}\left|b_{\alpha}(z)\right|=0
$$

Proof. If the functions $b_{\alpha}, 0 \leqslant|\alpha| \leqslant m$, are as in (4.1) and $0<r<1$, we define, for all $\alpha$, the compactly supported functions

$$
b_{\alpha, r}(z)= \begin{cases}b_{\alpha}(z) & \text { if }|z| \leqslant r \\ 0 & \text { if }|z|>r\end{cases}
$$

We also define the compactly supported distribution

$$
a_{r}=\sum_{0 \leqslant|\alpha| \leqslant m}(-1)^{|\alpha|} D^{\alpha} b_{\alpha, r}
$$

where the derivatives are again distributional. By Proposition 4.1, the Toeplitz operator $T_{a_{r}}: A^{p} \rightarrow A^{p}$ is compact for every $r$. On the other hand, due to the definition (2.9), the property (4.1) and the norm estimate (3.2), the operator norm

$$
\left\|T_{a}-T_{a_{r}}: A^{p} \rightarrow A^{p}\right\|=\left\|T_{a-a_{r}}: A^{p} \rightarrow A^{p}\right\|
$$

can be made arbitrarily small choosing $r$ close enough to 1 . Hence, $T_{a}$ must be a compact operator. 
Returning to the example (3.6), let us redefine the functions $b_{(0,0)}$ and $b_{(1,0)}$ by

$$
b_{(0,0)}(z)= \begin{cases}0 & \text { if } x \leqslant 0 \\ 2 c x\left(1-r^{2}\right)^{c-1} & \text { if } x>0\end{cases}
$$

and

$$
b_{(1,0)}(z)= \begin{cases}0 & \text { if } x \leqslant 0, \\ \left(1-r^{2}\right)^{c} & \text { if } x>0,\end{cases}
$$

where $c>1$ is arbitrary. Again, the symbol is a weighted Dirac measure of the line segment $\{z \in \mathbb{D} \mid \operatorname{Re} z=0\}$ with non-compact support. Moreover, in this case the resulting Toeplitz operator $A^{p} \rightarrow A^{p}$ is even compact.

Acknowledgements. A.P. and J.T. acknowledge the support from the Väisälä Foundation of the Finnish Academy of Science and Letters. J.V. was supported by a Marie Curie International Outgoing Fellowship within the Seventh European Community Framework Programme.

\section{References}

1. R. A. Adams, Sobolev spaces (Academic Press, 1975).

2. A. Alexandrov and G. Rozenblum, Finite rank Toeplitz operators: some extensions of D. Luecking's theorem, J. Funct. Analysis 256 (2009), 2291-2303.

3. S. Axler And D. Zheng, Compact operators via the Berezin transform, Indiana Univ. Math. J. 47 (1998), 387-400.

4. J. HoRvath, Topological vector spaces and distributions, Volume I (Addison-Wesley, Reading, MA, 1966).

5. A. Karapetyants, The space $\mathrm{BMO}_{\lambda}^{p}(\mathbb{D})$, compact Toeplitz operators with $\mathrm{BMO}_{\lambda}^{1}(\mathbb{D})$ symbols on weighted Bergman spaces, and the Berezin transform, Izv. VUZ Mat. 8 (2006), $76-79$.

6. W. Rudin, Functional analysis (McGraw-Hill, 1973).

7. D. SuAREZ, The essential norm of operators in the Toeplitz algebra on $A^{p}\left(\mathbb{B}_{n}\right)$, Indiana Univ. Math. J. 56 (2007), 2185-2232.

8. J. TASkinen And J. A. Virtanen, Spectral theory of Toeplitz and Hankel operators on the Bergman space $A^{1}$, New York J. Math. 14 (2008), 305-323.

9. J. Taskinen and J. A. Virtanen, Toeplitz operators on Bergman spaces with locally integrable symbols, Rev. Mat. Ibero. 26(2) (2010), 693-706.

10. N. VAsilevski, Commutative algebras of Toeplitz operators on the Bergman space, Operator Theory: Advances and Applications, Volume 185 (Birkhäuser, 2008).

11. K. ZHu, Positive Toeplitz operators on weighted Bergman spaces of bounded symmetric domains, J. Operat. Theory 20 (1988), 329-357.

12. K. ZHU, BMO and Hankel operators on Bergman spaces, Pac. J. Math. 155 (1992), 377-395.

13. K. ZHu, Operator theory in function spaces, 2nd edn, Mathematical Surveys and Monographs 138 (American Mathematical Society, Providence, RI, 2007).

14. N. Zorboska, Toeplitz operators with BMO symbols and the Berezin transform, Int. J. Math. Math. Sci. 46 (2003), 2929-2945. 